\title{
A construção do processo de trabalho no Núcleo de Apoio à Saúde da Família: a experiência dos farmacêuticos em um município do sul do Brasil
}

\author{
Construction of the work process of the Family Health Support \\ Nucleus: the experience of pharmacists in a city \\ in the south of Brazil
}

Carina Akemi Nakamura ${ }^{1}$

Silvana Nair Leite ${ }^{1}$
${ }^{1}$ Programa de Pós-

Graduação em Farmácia,

Universidade Federal de

Santa Catarina. Campus

Universitário Reitor João

David Ferreira Lima,

Trindade. 88040-970

Florianópolis SC Brasil.

silvana.nair.leite@ufsc.br
Abstract The Family Health Support Nucleus (NASF) was created in 2008 with the objective of broadening the range and scope of primary healthcare. The insertion of pharmacists in this multi-professional context represents an opportunity to enhance the working process and the rational access and use of medicines. The working processes of pharmacists in a city NASF was investigated. Field research was conducted using a qualitative approach with participant observation and semi-structured interviews. Two analytic dialectic categories emerged. The first was the pharmacists' dilemma in the construction of their working process as promoters of primary healthcare, while at the same time facing the need to deal with managerial functions mostly to cater to operational demand. The second was the reality experienced with guidelines and coordination of their work, where pharmacists can be free to structure their work as supporters, although at the same time it limits them due to lack of acknowledgment of their previously established working process. The lack of planning and a clear objective for work in the NASF, besides the deficiency of pharmaceutical services in primary healthcare make the development of any type of pharmacist activity important and essential even if it does not fully attend the NASF proposal.

Key words Primary health care, Pharmacist, Family health
Resumo Em 2008 foi criado o Núcleo de Apoio à Saúde da Família (NASF) com o objetivo de ampliar a abrangência e o escopo da atenção básica. A inserção do farmacêutico neste contexto multiprofissional representa uma oportunidade no seu processo de trabalho e no acesso e no uso racional de medicamentos. Investigou-se o desenvolvimento do processo de trabalho dos farmacêuticos nos NASF de um município. Foi realizada uma pesquisa de campo qualitativa por observação participante e entrevistas semiestruturadas. Duas categorias analiticas dialéticas emergiram: o dilema do farmacêutico na construção do processo de trabalho, como apoiador da atenção básica, mas que também lhe aprisiona em funções técnicogerenciais atendendo as demandas operacionais; e outra representando a realidade vivenciada na sua relação com as diretrizes e a coordenação do trabalho, tendo ao mesmo tempo "liberdade" para estruturar as tarefas como apoiador, mas que impõe limitaçães pelo pouco reconhecimento do processo laboral construído. Conclui-se que a falta de planejamento e de objetivo claro para os NASF e a deficiência de serviços farmacêuticos na atenção básica tornam o desenvolvimento de qualquer atividade por este profissional importante e necessária, mesmo não atendendo integralmente a expectativa da proposta do programa.

Palavras-chave Atenção Primária à Saúde, Farmacêutico, Saúde da família 


\section{Introdução}

O Núcleo de Apoio à Saúde da Família (NASF) foi criado em 2008 pelo Ministério da Saúde (MS) como uma estratégia inovadora, objetivando a ampliação da abrangência e o escopo das ações da Atenção Básica (AB), bem como sua resolutividade ${ }^{1,2}$. Constituído por equipes compostas por profissionais de diferentes formações, $\mathrm{o}$ NASF atua em parceria com os profissionais das equipes de saúde da família (SF), compartilhando as práticas em saúde e atuando diretamente no apoio dessas equipes nos territórios sob sua responsabilidade ${ }^{1}$.

A corresponsabilização entre NASF e Estratégia de SF acontece por meio do apoio matricial, que objetiva assegurar a retaguarda especializada, tanto assistencial quanto suporte técnico-pedagógico a equipes e profissionais encarregados da atenção à saúde, buscando construir e ativar a comunicação e o compartilhamento de conhecimentos entre os profissionais ${ }^{3,4}$. Esse apoio matricial se concretiza por meio de espaços de educação permanente, discussão de casos e atendimentos conjuntos, construção coletiva de planos terapêuticos e gestão de serviços, grupos compartilhados entre apoiadores e equipe de SF, intervenções conjuntas no território e ações intersetoriais e atendimentos específicos do apoiador, quando necessário ${ }^{5}$.

Os farmacêuticos estão entre os profissionais que podem compor as equipes do NASF, estando presente em cerca de $40 \%$ delas. Apesar disso, e da estratégia ter completado cinco anos da sua criação e três da publicação do documento "Diretrizes do NASF", estudos como o de Arioli ${ }^{6}$ demonstram ainda a falta de conhecimento dos profissionais das equipes de SF, do NASF e dos próprios farmacêuticos sobre as atividades a serem desenvolvidas em uma equipe multiprofissional, comprometendo seu papel e a visão dos demais sobre sua função na saúde da população.

Nesse momento de consolidação da proposta de contar com apoio referencial de equipes especializadas na $\mathrm{AB}$, de aumento da abrangência e de reformulação das ações para seu aprimoramento, além do reconhecimento e análise das experiências de implantação do NASF e da atuação do farmacêutico, buscou-se, através deste estudo, investigar a construção do processo de trabalho dos farmacêuticos que atuam nos NASF de um município do sul do Brasil.

\section{Metodologia}

Trata-se de um estudo de caso de abordagem qualitativa que utilizou como principal metodologia para a coleta de dados a observação participante ${ }^{7,8}$. O acompanhamento ocorreu entre março e dezembro de 2012, por cerca de 10 dias de trabalho de cada um dos 7 farmacêuticos que atuavam nas 7 equipes cadastradas no município. Durante este período, todas as atividades profissionais dos farmacêuticos (reuniões de equipe, visitas domiciliares, períodos nas unidades de saúde, reuniões de farmacêuticos, atividades de grupo, confecção de relatórios, atendimentos individuais e muitos outros) foram acompanhadas pela pesquisadora, que se utilizou do ouvir, escutar, ver, fazer uso de todos os sentidos para apreender o fazer desses profissionais, sua inserção e interação com as equipes e com os usuários, incluindo também suas criações e frustrações durante o processo de trabalho. Todas as observações, incluindo falas e expressões de outros profissionais e de usuários, foram registradas em diário de campo.

Entrevistas semiestruturadas foram aplicadas com todos os farmacêuticos ${ }^{9,10} \mathrm{em}$ momentos oportunos, com o objetivo de esclarecer processos observados e permitir reconhecer as opiniões e as expectativas dos profissionais sobre seus processos de trabalho. As falas ficaram anônimas e os farmacêuticos foram identificados pela letra $\mathrm{F}$ seguida de um número de 1 a 7 . Todo o material de campo foi analisado pelo conteúdo ${ }^{11}$, seguindo o roteiro de análise: familiarização com o texto completo de todas as entrevistas e observações; identificação de todos os conceitos e temas relevantes; indexação dos trechos destacados por tema e correlação com outros; rearranjo dos trechos destacados, considerando seus contextos e peculiaridades, em um fluxo coerente e compreensível; mapeamento dos conceitos, abrangência e natureza do fenômeno estudado; busca pela associação dos temas para a construção de uma explanação para os achados, ligando-os ao referencial teórico ${ }^{12}$, buscando a interpretação dos sentidos do observado como resultado ${ }^{10}$.

O estudo ocorreu em um município do sul do Brasil cuja população é de cerca de $400 \mathrm{mil}$ habitantes e a rede pública de saúde dividida em 50 Centros de Saúde (CS) e sete equipes do NASF, sendo um farmacêutico para cada equipe. O estudo foi aprovado pelo Comitê de Ética em Pesquisa com Seres Humanos da Universidade Federal de Santa Catarina. 


\section{Resultados}

\section{Os Farmacêuticos e seu processo de trabalho nos NASF}

Os sete farmacêuticos participantes estavam atuando no NASF entre 5 meses e 2 anos e meio, sendo responsáveis por de 5 a 11 CS e de 12 a 24 equipes de SF. Ressalta-se que quatro dos farmacêuticos tinham especialização em SF, sendo que dois a realizaram na forma de Residência Multiprofissional em Saúde da Família (RMSF), anteriormente ao início do trabalho no NASF e dois começaram a especialização posteriormente ao início das suas atividades no NASF.

Em 2009, sete NASF estavam implantados no município. No início da implantação eram realizadas reuniões quinzenais com todos os profissionais para organização do processo de trabalho, já para os profissionais que começaram atuar posteriormente, não houve capacitação formal, apenas a participação pontual em algumas (poucas) reuniões do NASF. Segundo esses profissionais, a preparação foi de forma individual ou conhecendo o trabalho de colegas que já atuavam no NASF.

Mesmo após três anos da inserção dos farmacêuticos nos NASF no município, foi relatado pelos profissionais que o processo de trabalho ainda não está bem definido e estruturado. Ao iniciar suas atividades no NASF, os profissionais puderam elaborar e organizar seus próprios cronogramas, tendo, de forma geral, distribuído suas 40h/semanais com um grande número de CS e de equipes de SF sob sua responsabilidade, como pode ser observado nos relatos a seguir:

[F4]: Quando eu assumi, tive que definir como iria distribuir minha carga horária, como que minimamente eu ia me organizar, foi um processo pessoal, não teve uma construção coletiva nem com a coordenação do distrito, só me pediram pra fazer uma proposta de como seria a minha organização, mas fui eu que sentei e elaborei uma proposta.

[F3]: Foi definido de acordo com a necessidade da unidade, o que a unidade queria, [você] se adapta e faz o trabalho. Se uma unidade precisa do farmacêutico na dispensação, é feita dispensação e capacitação do técnico, se precisa de ajuda em grupos $[\ldots]$.

[F7]: Eu procurei priorizar algumas coisas, como era trabalho demais pra uma pessoa só, eu procurei priorizar aquilo que geraria mais impacto, então eu procurei priorizar algumas unidades que tinham mais abertura para o trabalho, aquelas unidades que eram mais resistentes eu deixei mais de lado.
Dessa forma, os farmacêuticos aplicam em seus cronogramas critérios de demandas criadas nos CS, juntamente como tipos de atividades que desejam realizar e experiências que têm para trabalhar na lógica do NASF, de formas variadas.

Em 2010 foi realizada a primeira oficina dos NASF para debater sua atuação, discutir o processo de trabalho como um todo, além de temas como o matriciamento e as dificuldades encontradas. Apenas em 2012 começaram a ser realizadas reuniões por categoria profissional para que fosse definido o processo de trabalho de cada uma. Como resultado dessas reuniões foi definido o cronograma, que prevê atividades mínimas que o profissional deve realizar e suas respectivas cargas horárias mínimas, sendo função de cada farmacêutico organizar suas tarefas mensais.

$\mathrm{Na}$ construção do cronograma, para os farmacêuticos sem experiência prática na lógica da SF, o trabalho dentro da farmácia apresentou-se como a melhor forma de criação de vínculo com as equipes e os CS. Já para os farmacêuticos que fizeram a RMSF, houve uma maior facilidade na realização de atividades assistenciais, envolvendo as equipes de $\mathrm{SF}$ e os usuários.

Durante o período de acompanhamento dos farmacêuticos, a dispensação de medicamentos nas farmácias nos CS foi a atividade realizada com maior frequência e por todos os eles, porém em curtos períodos de tempo em diversos CS, o que inviabiliza a criação de vínculo e a referência do serviço. Foi seguida da participação em grupos de educação em saúde, realizada com maior frequência e pela requisição de medicamentos para abastecimento das farmácias, realizada pela maioria dos farmacêuticos.

\section{Categorias analíticas}

Após a coleta e a observação dos dados e a descrição do perfil do processo de trabalho dos farmacêuticos, a análise e a interpretação de todos os achados em campo resultou na construção de categorias analíticas com base dialética. Nessa lógica, emergiram dois grupos de categorias: uma representando o dilema do profissional farmacêutico na definição das atividades a serem realizadas, ora sendo um apoiador das equipes de SF, ora sendo um farmacêutico mais restrito às farmácias; e outra, representando a realidade enfrentada pelos profissionais na definição de seu processo de trabalho, tendo ao mesmo tempo uma "liberdade" para estruturá-lo como apoiador da equipe de SF e as limitações impostas pelo cotidiano dos serviços de saúde. As categorias 
analíticas assim definidas são apresentadas a seguir.

\section{Dilemas na construção do processo de trabalho: apoiador matricial das equipes de SF?}

A oportunidade de se inserir em uma equipe multiprofissional para o desenvolvimento das atividades foi citado como positivo por muitos dos farmacêuticos, já que permite a troca de conhecimentos com outros profissionais, qualificando e potencializando o trabalho, além de permitir a participação em diversas áreas fora do campo de atuação individual da farmácia. Porém, foi observado que as atividades em conjunto com as equipes de SF ainda compreendem uma pequena parte do cronograma mensal do farmacêutico. Assim como citado por Vannucchi e Carneiro Junior $^{13}$ e Arioli ${ }^{6}$ e também observado na realidade do município, ainda não há uma compreensão completa do trabalho do NASF, tanto pelos seus profissionais quanto para as equipes de SF. A sobrecarga de trabalho dos profissionais dos NASF é um dos fatores identificados por Hori e Nascimento $^{14}$ para a dificuldade de percepção de "equipe em integração", que compartilha experiências e conhecimentos e colabora mutuamente. Os profissionais, segundo os autores, acabam por reproduzir práticas tecnicistas e de encaminhamentos. Já o documento com as diretrizes do $\mathrm{NASF}^{1}$, que deveria ser referência para o trabalho, acabou por ser um dicionário de conceitos-chave, definindo-os teoricamente na maior parte do tempo, ou como citado por Vannucchi e Carneiro Junior $^{13}$, o documento diz "o que fazer", mas não "como fazer". A versão publicada em 2014 dos Cadernos de Atenção Básica ${ }^{15}$ coloca um direcionamento mais claro, interdisciplinar e organizador dos processos de trabalho para as equipes do NASF e pode vir a trazer maior segurança no desenvolvimento deste processo. Pereira ${ }^{16}$ relata a falta de domínio dos trabalhadores do NASF quanto ao seu processo de trabalho, especialmente para as atividades não tecnicamente definidas para sua profissão, referentes à interdisciplinaridade, ao trabalho em equipe e ao matriciamento. Para a autora, esta insegurança contribui para uma práxis conservadora e alienada.

Segundo o documento Diretrizes do NASF${ }^{1}$, a atuação dos profissionais é baseada em uma tecnologia de gestão chamada apoio matricial. Contudo, observou-se que a relação das equipes de SF com os farmacêuticos não acontece exatamente nesta perspectiva, pois muitas vezes não ocorre o matriciamento, fato também relatado por F3: “[...] um fluxo que deveria ser via matriciamento e não acontece”. Os casos nem sempre são referenciados das equipes de SF para o farmacêutico e vice-versa, estabelecendo assim um novo fluxo de acesso ao farmacêutico do NASF, em que a proximidade com os usuários durante a dispensação ou em grupos de educação em saúde permite que o usuário tenha acesso ao serviço farmacêutico de uma forma direta, mas sem regularidade ou planejamento deste acesso ao profissional. Além disso, os demais farmacêuticos da rede municipal também podem ser pontes de acesso ao serviço, já que as relações pessoais e profissionais estabelecidas pelo farmacêutico do NASF permitem que os casos observados por outros profissionais possam ser encaminhados diretamente para ele, sem um fluxo estabelecido ou regular de matriciamento.

Uma espécie de matriciamento observado durante o acompanhamento dos farmacêuticos e também citado por Arioli ${ }^{6}$ são as discussões e elaborações de projetos terapêuticos realizados em situações informais, como as "conversas de corredor”, envolvendo poucos profissionais e de acordo com as necessidades e da proximidade entre os profissionais e não nos espaços das reuniões. Sendo assim, confirma-se a teoria de Vannucchi e Carneiro Junior ${ }^{13}$ de que as ações visando à resolução dos casos estão acontecendo, independente da forma como estão sendo executadas (via matriciamento ou não), fruto da ausência de metodologias nos documentos orientadores do programa. Assim, mesmo sem estar funcionando como a proposta, o NASF já mostra sua importância e pontos positivos, como citado por um farmacêutico que acredita que trabalhando nessa lógica, pode conhecer a história de vida dos usuários, gerando mudanças na abordagem do atendimento, diferentemente do trabalho executado isoladamente nas farmácias.

É possível observar na prática que ao trabalhar com uma equipe mais estruturada em relação à lógica da SF e dos serviços do próprio CS, incluindo a farmácia, o papel do farmacêutico como equipe de NASF é muito mais evidente, diferentemente do que ocorre em CS com equipes não estruturadas, em que o farmacêutico do NASF, basicamente, exerce atividades gerenciais desconectadas do cuidado e limitado ao espaço da farmácia.

Além disso, a deficiência de formação específica para trabalhar nessa nova lógica, a falta de experiência e, em muitos casos, o desinteresse dos profissionais das equipes de SF e do próprio NASF em trabalhar de forma interdisciplinar 
acabam impedindo o funcionamento do NASF conforme o esperado.

\section{Dilemas na construção do processo de trabalho: atendimento das demandas por serviços farmacêuticos?}

Se por um lado a possibilidade de atuar na assistência e na gerência gera certa angústia para alguns profissionais por ter que optar pelas atividades a serem desempenhadas, por outro, pode "engessar" 17 o processo de trabalho, já que permite ao farmacêutico focar em atividades com as quais se sinta mais confortável, não necessariamente na lógica de trabalho proposta para o NASF ou das reais necessidades das equipes. A farmácia se apresentou como a melhor forma de criar vínculos com os profissionais das equipes de SF, devido à associação farmácia-farmacêutico, e podendo, ainda, ser considerada o seu "cantinho" pelos farmacêuticos e pelos demais profissionais. Além disso, soma-se o fato de as farmácias ficarem sob a responsabilidade "de todos e de ninguém ao mesmo tempo" ${ }^{18}$, e as equipes dos CS esperarem pela assistência de um profissional qualificado, sendo o farmacêutico bem-vindo para "cuidar da farmácia".

A falta de profissionais das equipes de SF também se apresenta como um dificultador, sendo que pouquíssimas foram as equipes completas observadas. Os técnicos de enfermagem são responsáveis por diversas atividades em diferentes setores, assim, na ausência de profissionais e na presença de farmacêutico, torna-se viável e lógico trabalhar em outra atividade que necessite de apoio, deixando a farmácia para o farmacêutico, sem auxiliares. Foi citado por equipes de SF, por diversas vezes, o interesse em ter o farmacêutico no CS, mais precisamente dentro da farmácia, todos os dias, demonstrando que esta é a atividade esperada para este profissional (e uma demanda real não atendida pela organização da $\mathrm{AB}$ ), mais que a função de matriciamento.

Como último fator, e talvez o mais relevante, por ser preocupante, tem-se a influência da formação do profissional no processo de trabalho. Os cursos de graduação não têm formado profissionais para trabalhar nessa nova lógica, confirmado também em pesquisa realizada por Silva et al. ${ }^{17}$. Somado a isso, a insegurança e o medo do farmacêutico para atuar fora da farmácia, a falta de habilidades e capacitação para novas atividades, além do não reconhecimento do seu papel fora da farmácia por ele próprio, pelas equipes de SF e pelos usuários podem levar os farmacêuticos a se prenderem à farmácia como espaço e como atividade, limitando o fazer, a criação e os resultados em saúde esperados pela proposição dos NASF.

\section{As realidades do processo de trabalho: uma possível "autonomia"}

A "liberdade" para definição de suas agendas, permitida ao profissional pela coordenação regional das equipes de saúde do município, é a chave para o desenvolvimento de certa autonomia para a construção do processo de trabalho do farmacêutico no NASF. O profissional pode distribuir sua carga horária de trabalho entre as diversas atividades nos diferentes CS, sendo influenciado, principalmente, pela sua formação acadêmica e percepções pessoais sobre as atividades que quer ou precisa desenvolver. Por ser uma proposta considerada nova, o seu não entendimento por completo, tanto pelas equipes de SF quanto pelos próprios profissionais do NASF, torna qualquer atividade resolutiva sinônimo de um bom trabalho.

A "liberdade" dada por parte da gerência, não prescrevendo tarefa, execuções e horários tem permitido, para parte dos farmacêuticos pesquisados (preparados e dispostos), que o trabalho do profissional seja um trabalho vivo. Este ocorre no momento em que está sendo produzido, referindo-se ao uso de saberes e práticas próprios da profissão, juntamente ao seu potencial inventivo e criativo ${ }^{19}$. Exemplo disso é a ausência de rotina e a imprevisibilidade, pois diferentes indivíduos e diferentes casos surgem a todo o momento ${ }^{6}$. Além disso, nem sempre o que foi planejado se concretiza, necessitando da habilidade do profissional para reorganizar suas atividades e dando a ele autonomia para gerir as ferramentas a partir dos dados e dos saberes que domina ${ }^{20}$. Traz-se, ainda, a ideia do trabalho real, resultado das situações reais de trabalho que são dinâmicas, instáveis e submetidas a imprevistos, não havendo condições ideais ou idealizadas ${ }^{21}$.

No entanto, a mesma "autonomia" para definir sua agenda e forma de trabalho, faz do processo de trabalho também um resultado mais do "acaso" do que de planejamento e organização. Muitas vezes, o usuário consegue resoluções para seus casos ao entrar em contato direto com o farmacêutico, independente do contato prévio com a equipe de SF. Entretanto, nada garante que outro usuário ou o mesmo obteria a mesma resolubilidade para o mesmo caso se tivesse a ajuda de outro profissional ou se não contasse com a sorte de encontrar o farmacêutico no CS. Os casos observados remetem à reflexão sobre como estas 
portas de entrada aleatórias podem ser consideradas reais e efetivas, se geram acolhimento e resolubilidade muito diferentes de caso para caso, sem garantias e continuidades, por serem irregulares e dependentes das características e relações pessoais de cada profissional.

Campos $^{22}$ argumenta que, em geral, os métodos de gestão tendem a reduzir o grau de autonomia dos trabalhadores, por formas diretas ou indiretas de controle do trabalho e, na saúde, a decisão autônoma do trabalhador é fundamental por suas características de imprecisão e singularidade - por tratar-se do trabalho vivo em ato. Neste cenário, no entanto, é pertinente questionar até que ponto o que ocorre é autonomia no processo de trabalho para os farmacêuticos do NASF e o quanto há de ingerência e falta de clareza de objetivos para a proposta do NASF como um todo e para o profissional farmacêutico como componente desta estratégia de organização de trabalho na $\mathrm{AB}$.

A autonomia técnica, no entanto, é mais frequente na observação do cotidiano das atividades dos farmacêuticos. No seu fazer específico o farmacêutico demonstra melhor preparo e iniciativa e é melhor compreendido por seus colegas de trabalho. Para atividades de equipe, como matriciamento e discussões conjuntas de casos, muitas vezes o farmacêutico é limitado em sua autonomia de participar de forma equânime nas decisões, pois não é normalmente reconhecido como parte do processo clínico. Em reuniões observadas, os farmacêuticos com melhor formação em SF demonstraram iniciativa para esta participação, chamando para si a responsabilidade de participar das intervenções sobre os casos discutidos, mas ainda assim, de forma muito dependente de suas características individuais e não como uma característica comum a esses profissionais do NASF.

\section{A realidade do processo de trabalho: diversas formas de limitação}

Se, por um lado, o trabalho no NASF tem aspectos autônomos, por outro, pode ser limitador, pois as mesmas coordenações que não interferem no cronograma de trabalho cotidiano, tratam o farmacêutico do NASF como um "tapa buracos", exigindo sua presença na substituição de qualquer colega de profissão de um centro de referência ou serviço que dispense medicamentos da Portaria 344/98.

O farmacêutico organiza seu cronograma mensal no final do mês anterior, incluindo as atividades pactuadas anteriormente com as equipes de SF. Fato nada raro é o recebimento de ligações da gerência convocando-o para substituir um farmacêutico de um serviço de saúde no dia seguinte ou no período seguinte, necessitando assim desmarcar compromissos agendados nos CS, que nunca são prioridade na organização geral do funcionamento da rede de atenção. Algumas coordenações aparentam ser mais flexíveis no caso do farmacêutico não conseguir desmarcar as atividades agendadas anteriormente, porém, outras tratam a substituição como uma obrigação - função precípua de cobrir os horários e locais onde outros colegas (não definidos como parte dos NASF) estejam faltando. Observou-se ainda que o farmacêutico do NASF é a primeira opção de substituição para a coordenação, que não utiliza como alternativa um remanejamento dos horários de trabalho desses profissionais das farmácias de referência para evitar a irregularidade e a concretude do processo de trabalho. Esse fato tem sido considerado uma das maiores barreiras para a estruturação do processo de trabalho pelos farmacêuticos, como observado no relato de um deles.

[F5]: O grande dificultador é falta de $R H$ [recursos humanos], ter que cobrir outros profissionais e isso quebra o processo de trabalho [...]. O farmacêutico tira férias, entra em licença, pega atestado, o farmacêutico do NASF que vai cobrir e prejudica muito o processo de trabalho, ele é muito frágil ainda, o farmacêutico volante faz parte do processo de trabalho.

Além das limitações impostas pela gerência das equipes, o próprio farmacêutico também pode limitar seu processo de trabalho quando impede a flexibilização do seu cronograma mensal, caso observado nos cronogramas pré-definidos, em que definem os CS que irão apoiar em cada período da semana, se mantendo igual a cada semana por diversos meses. Dessa forma, se ocorre uma reunião de equipe ou uma atividade comunitária nos CS, o farmacêutico é "impedido" de participar das diferentes atividades, pois seu cronograma indica que ele estará em determinado CS naquele dia. No caso de não haver atividades nesse CS, mas em outro sim, o farmacêutico continua seguindo seu cronograma, com algumas exceções pontuais, como um convite para participar de um grupo. Como já observado por Galavote et al..$^{23}$, muitas vezes o trabalho dos profissionais pode estar centrado mais na esfera institucional do que na solidária e social, estando o profissional mais preocupado em cumprir sua carga horária do que em desenvolver atividades durante o período de trabalho. 
Além disso, os farmacêuticos, assim como os demais profissionais das equipes de SF, podem trabalham seguindo fielmente as normativas, fato também citado por Sartor ${ }^{18}$, produzindo trabalho morto, nas palavras de Merhy e Franco ${ }^{24}$. Os problemas encontrados durante a dispensação, como receitas médicas apresentando alguma irregularidade, por exemplo, podem impedir que o usuário receba o medicamento, interrompendo assim seu tratamento, apenas por ferir as normativas. Observa-se a necessidade de mudar o foco da receita médica para o usuário durante a dispensação, pois fatos como esse levam o usuário a desacreditar no serviço de saúde como ambiente de acolhimento e resolução de suas necessidades, além de se sentir ou ser induzido a se sentir responsabilizado e sofrer as consequências de um erro que, muitas vezes não é seu.

A ausência de reconhecimento do papel do farmacêutico como profissional de saúde pelas equipes de SF e do NASF, ao mesmo tempo em que lhe permite criar e inovar, é considerada um limitante, quando estas não conseguem ver as potencialidades de suas ações na resolução de casos. Para conseguir realizar seu trabalho, os farmacêuticos precisam oferecer seus serviços e solicitar sua participação nos casos clínicos, ou ficar limitado às funções técnico-gerenciais da farmácia.

\section{Conclusões}

Conclui-se que a falta de conhecimento do papel e das funções dos farmacêuticos podem representar a condição das equipes de SF e deles próprios, por ser uma nova lógica implantada no SUS, devido à deficiência de formação específica para a utilização de tecnologias leves e pela falta de orientação adequada aos profissionais ao iniciarem no NASF. Observa-se que a proposta do NASF para os farmacêuticos carece ainda de objetivos claros, impedindo o planejamento (por não saber aonde se quer chegar) e, consequentemente, a definição do processo de trabalho. Essa carência, juntamente com a de serviço farmacêutico básico na rede de $\mathrm{AB}$ como um todo, acabam tornando qualquer atividade realizada pelo profissional sinônimo de um bom serviço, independente de estar relacionada ao matriciamento ou não. Qualquer atividade não é necessariamente o que se espera do NASF, porém, no caso do farmacêutico se espera tudo, ou seja, qualquer coisa, já que é impossível que todas as atividades citadas e descritas nas Diretrizes do NASF para a Assistência Farmacêutica sejam realizadas por um profissional que se responsabilize por diversas equipes de SF e CS.

Ao mesmo tempo em que há certa "liberdade" e "autonomia", por parte da gerência, para organização do seu trabalho, essa falta de gerenciamento é considerada limitadora, pois uma das premissas da autonomia é agir sabendo o que deve fazer e, no caso do farmacêutico no NASF, os objetivos, as metas e os resultados a serem alcançados ainda não estão claros o suficiente para os próprios profissionais e para a gerência. Assim, o farmacêutico deve construir seu processo de trabalho, buscando criar, inovar, contribuir para a saúde, buscar inter-relações com outros profissionais e desenvolver ações intersetorais para que seja respeitado e resolutivo, evitando que se torne um “tapa buracos”. É necessário, ainda, que o farmacêutico perceba o resultado do seu trabalho, conseguindo se observar e observar suas ações nele, o que contribuiria para o surgimento e fortalecimento do sentimento de responsabilidade e compromisso. Observa-se, aqui, a ambiguidade do matriciamento também, pois ele tem características e potência para a construção do processo de cuidado em saúde, mas também possui armadilhas, sendo uma delas a ausência ou diminuição do contato direto com o usuário, impedindo assim que o resultado do seu trabalho seja observado, já que o objeto dessa ferramenta passa a ser a equipe de SF.

Essa ausência de objetivo claro pode também ser percebida por outros profissionais, que podem esperar que o farmacêutico trabalhe apenas dentro da farmácia. Por outro lado, pode não haver expectativas sobre seu trabalho, o que permite que ele busque participar do matriciamento, dos grupos de educação em saúde, de visitas domiciliares, apoiar as equipes de SF na parte gerencial e assistencial, desenvolvendo o seu processo de trabalho na prática, na busca de seu objetivo final. Assim, essa nova lógica na presença de farmacêuticos preparados, com formação específica, dispostos, com compromisso e proatividade, pode possibilitar a construção de uma nova história do fazer farmacêutico na $\mathrm{AB}$, mas, para tanto, necessita de clareza de objetivos, de metas, de responsabilidades e compreensão mais ampla do papel do trabalho em equipe no contexto da atenção à saúde. 


\section{Colaboradores}

CA Nakamura trabalhou na elaboração do projeto, coleta e análise dos dados e redação do manuscrito e SN Leite na elaboração do projeto, análise dos dados, redação do manuscrito e na aprovação final.

\section{Referências}

1. Brasil. Ministério da Saúde (MS). Secretaria de Atenção à Saúde. Departamento de Atenção Básica. Diretrizes do NASF: Núcleo de Apoio a Saúde da Família. Brasília: MS; 2010.

2. Molini-Alvejonas DR, Mendes VLF, Amato CAH. Fonoaudiologia e Núcleos de Apoio à Saúde da Família: conceitos e referências. Rev Soc Bras Fonoaudiol 2010; 15(3):465-474.

3. Campos GWS. Equipes de referência e apoio especializado matricial: um ensaio sobre a reorganização do trabalho em saúde. Cien Saude Colet 1999; 4(2):393-403.

4. Campos WSC, Domitti AC. Apoio matricial e equipe de referência: uma metodologia para gestão do trabalho interdisciplinar em saúde. Cad Saude Publica 2007; 23(2):399-407.

5. Arona EC. Implantação do Matriciamento nos Serviços de Saúde de Capivari. Saúde Soc 2009; 18(1):26-36.

6. Arioli IGS. Práticas e estilos de pensamento em promoção da saúde no contexto da atenção básica [dissertação]. Florianópolis: Universidade Federal de Santa Catarina; 2012.

7. Maffezzolli ECF, Boehs CGE. Uma reflexão sobre o estudo de caso como método de pesquisa. Rev FAE 2008; 11(1):95-110.

8. Cartana MHF. El estúdio de caso em investigación en enfermería. In: Prado ML, Souza ML, Carraro TE, editores. Investigación Cualitativa en enfermería: Contexto y bases conceptuales. Washington: OPS, OMS; 2008. (Serie PALTEX Salud y Sociedad 2000, n.9). p. 213-221.

9. Yin RK. Estudo de caso. Planejamento e Métodos. $3^{\mathrm{a}} \mathrm{ed}$. Porto Alegre: Bookman; 2005.

10. Minayo MCS. O desafio do conhecimento. Pesquisa qualitativa em saúde. $12^{\mathrm{a}}$ ed. São Paulo, Rio de Janeiro: Hucitec, Abrasco; 2010.

11. Bardin L. Análise de Conteúdo. Lisboa: Edições 70; 2010.

12. Pope C, Zieblnad S, Mays N. Qualitative research in healthcare: analyzing qualitative data. BMJ 2000; 320(7227):114-116.

13. Vannucchi AMC, Carneiro Junior N. Modelos tecnoassistenciais e atuação do psiquiatra no campo da atenção primária à saúde no contexto atual do Sistema Único de Saúde, Brasil. Physis 2012; 22(3):963-982.

14. Hori AA, Nascimento AF. O Projeto Terapêutico Singular e as práticas de saúde mental nos Núcleos de Apoio à Saúde da Família (NASF) em Guarulhos (SP), Brasil. Cien Saude Colet 19(8):3561-3571.

15. Brasil. Ministério da Saúde (MS). Secretaria de Atenção à Saúde. Departamento de Atenção Básica. Núcleo de Apoio à Saúde da Família. Volume 1. Ferramentas para a gestão e para o trabalho cotidiano. Brasília: MS; 2014. (Cadernos de Atenção Básica, n. 39).
16. Pereira KG. Conflitos éticos no processo de trabalho do Núcleo de Apoio à Saúde da Família em um município de Santa Catarina/SC [dissertação]. Florianópolis: Universidade Federal de Santa Catarina; 2011.

17. Silva ATC, Aguiar, ME, Winck K, Rodrigues KGW, Sato ME, Grisi SJFE, Brentani A, Rios IC. Núcleos de Apoio à Saúde da Família: desafios e potencialidades na visão dos profissionais da Atenção Primária do município de São Paulo, Brasil. Cad Saude Publica 2012; 8(11):20762084.

18. Sartor VB. A Assistência Farmacêutica e a Estratégia Saúde da Família: em busca da integralidade da atenção à saúde no curso de especialização em Saúde da Famílial modalidade Residência - UFSC [dissertação]. Florianópolis: Universidade Federal de Santa Catarina; 2004.

19. Merhy EE. Saúde: a cartografia do trabalho vivo. São Paulo: Hucitec; 2002.

20. Faria HX, Araujo MD. Uma perspectiva de análise sobre o processo de trabalho em saúde: produção do cuidado e produção de sujeitos. Saúde Soc 2010; 19(2):429-439.

21. Brito JC. Trabalho real. In: Pereira IB, Lima JCF, organizadores. Dicionário da Educação Profissional em Saúde [internet]. Rio de Janeiro: Fiocruz, 2009. 2 ed. [acesso em dez 2012]. Disponível em: http://www.epsjv.fiocruz.br/dicionario/Dicionario2.pdf.

22. Campos GWS. A mediação entre conhecimento e práticas sociais: a racionalidade da tecnologia leve, da práxis e da arte. Cien Saude Colet 2011; 16(7):3033-3040.

23. Galavote HS, Prado TN, Maciel ELN, Lima RCD. Desvendando os processos de trabalho do agente comunitário de saúde nos cenários revelados na Estratégia Saúde da Família no município de Vitória (ES, Brasil). Cien Saude Colet 2011; 16(1):231-240.

24. Merhy EE, Franco TB. Por uma Composição Técnica do Trabalho Centrada nas Tecnologias Leves e no Campo Relacional. Saúde em Debate 2003; 27(65):316-323.

Artigo apresentado em 18/07/2014

Aprovado em 22/08/2015

Versão final apresentada em 24/08/2015 